\title{
Towards a Reliable and Sensitive Deep Learning Based Approach for Multi-Ship Detection in SAR Imagery
}

\author{
Elshafey, M. A. \\ Department of Computer Engineering and Artificial Intelligence, Military Technical College, \\ Cairo, Egypt E-mail: m.shafey@mtc.edu.eg \\ DOI: https://doi.org/10.52939/ijg.v17i6.2067
}

\begin{abstract}
Synthetic Aperture Radar (SAR) images show promising results in monitoring maritime activities. Recently, Deep learning-based object detection techniques have impressive results in most detection applications but unfortunately there are challenging problems such as difficulty of detecting multiple ships, especially inshore ones. In this paper, a three-step ship detection process is described and a reliable and sensitive hybrid deep learning model is proposed as an efficient classifier in the middle step. The proposed model combines the finetuned Inception-Resnet-V2 model and the Long Short Term Memory model in two different approaches: parallel approach and cascaded approach. In experiments, the region proposal algorithm and the NonMaxima suppression algorithm are applied in the first and last step in the three-step detection process. The comparative results show that the proposed approach in cascaded form outperforms the competitive recent state-of-the-art approaches by enhancement up to 16.3\%, 16.5\%, and 18.9\% in terms of recall, precision and mean average precision, respectively. Moreover, the proposed approach shows high relative sensitivity for challenged cases of both inshore and offshore scenes by enhancement ratios up to $81.88 \%$ and $24.58 \%$, respectively in recall perspective.
\end{abstract}

\section{Introduction and Related Work}

Synthetic Aperture Radar (SAR) system is considered a perfect source of images (Chaturvedi, 2019 and Cui et al., 2021) specially for military applications, environmental monitor, marine monitoring, or any other application that requires a relatively high accuracy due to the fact that neither weather nor light can affect the imaging process of the SAR. With the increasing count of launching satellites, such as Sentinel-1, Gafon-3 and Terra SAR-X, the availability of SAR images has been increased. Maritime transport has an essential role in growth and sustainable development that leads to a significant increase in the currently ships count. The management of marine traffic is one of the most important applications of SAR images, from which ships can be detected easily due to their bright intensity (Cui et al., 2021 and Arivazhagan et al., 2019). Meanwhile, in real scenario, a SAR image contains other objects besides ships, which are not interested in, such as sea ice and coastline structure, and even speckle noise that may negatively affect the ship detection process (Song et al., 2017).

During the past decades, researchers were interested in the field of SAR ship detection and many researches have been published. In (Liang et al., 2020) Constant False-Alarm Rate (CFAR) algorithm is presented that is able to identify targets statistically by setting a threshold while maintaining a constant false alarm rate. In (Ai et al., 2010) a 2-D joint lognormal distribution algorithm, which uses the correlation of the gray intensity to model the ship targets is demonestrated. A new hierarchical scheme for detecting ships in SAR images is proposed in (Wang and Liu, 2012), which consists of detection and discrimination modules, such that ship candidates are obtained by applying CFAR, while ship discrimination is performed by using one-class classification. Another method in (Hou et al., 2013) is proposed to detect ship targets by measuring the visual conspicuity of each water region and the k-means clustering algorithm. In (Zhang et al., 2018) a ship segmentation scheme with non-local processing is proposed in order to handle the speckle noise and the complicated backscattering phenomenology in SAR images. These ship detection approaches are based on manually extracted features, experienced statistic model and traditional image processing methods. A prior knowledge is required, and optimization difficulty is another challenge.

In recent years, deep learning, which is able to automatically extract features, has encountered 
another revival especially with the rapid development of hardware computing (Fouad et al., 2020). An impressive work has been done to highlight the superiority of methods based on deep learning over traditional methods (Wang et al., 2018 and Carman and Kolhatkar, 2020). A lot of Convolutional Neural Network $(\mathrm{CNN})$ architectures has already achieved great success for image features extraction, classification tasks in computer vision field and region proposal-based object detectors. For instance, the transfer learning and single shot multibox detector (SSD) are combined, in (Wang et al., 2018), for ship detection. However, the SSD provides a relatively low accuracy. In (Kang et al., 2017), a faster region-based convolution neural network (R-CNN) is adapted to solve the ship detection problem with higher accuracy. However, the multi-scale characteristics of the SAR ship images are not considered. For instance, the approach in (Wang et al., 2019) based on RetinaNet (Lin et al., 2017), shows higher accuracy for large size ships, however with a small detection rate for small size ships. As well, in (Zhou et al., 2020), a multi-scale version of the Faster RCNN (Ren et al., 2016) is presented to solve the detection problem of multi scaled images and small sized ships, however, with a small detection rate for ships near the port, i.e., complex background. The problem of multi scale problem in SAR imagery are solved by proposed methods in (Liu et al., 2019) based on merging the strong scattering components and super-pixel grouping. These methods can achieve 0.85 of recall metric. In (Zhao et al., 2020), an attention-based model, is proposed to construct fine-grained feature pyramid. The proposed model can handle ships of various sizes with complex backgrounds based on combination of receptive filed and attention blocks.

In this paper, a hybrid Deep Learning (DL) approach, based on Convolution Neural Network (CNN) model and Long Short Term Memory (LSTM) model, is presented as a reliable and sensitive classifier to enhance the detection accuracy of the multi scaled small sized ships in the SAR images. In work, both the bagging method and boosting method, for obtaining more efficient DL hybrid approach, are discussed. By the bagging method, a proposed parallel approach is generated, while a proposed cascaded approach is generated by the boosting method. The two proposed approaches are evaluated and compared with recent state-of-the approaches. The paper is organized in 4 main sections. Section 2 explains, in detail, the three-step ship detection process, including the proposed hybrid DL approach. Experimental work and comparative analysis are illustrated in Section 3. Finally, conclusions and suggested future work are drawn in Section 4.

\section{Methodology}

Ship detection process, in SAR images, is a complex problem that has three main issues to be considered: i) the nature of multi-scale characteristics of ships in SAR images, ii) the difficulty of detecting ships in complex backgrounds, and iii) the challenge of detecting small ships. This section presents the detailed stages towards the proposed approach to deal with such issues and perform the detection task with a relatively high accuracy and reliability.

In order to perform ship detection, the input SAR image should pass through a three-step process, as shown in Figure 1. In the first step, a Regions of Interest (ROI) generation process is applied that accepts an input image and results proposals for the locations (ROIs) of the detected objects in the image. In the second step, the generated ROIs are classified using an efficient classifier to decide if the detected object in each ROI is a ship or not. In the last step, a process for eliminating overlapped bounding-boxes (if any) of the classified ships is applied to form a single detection bounding box (Pang et al., 2019).

\subsection{Region Proposal Algorithm for ROI Generation}

The most traditional methods that are commonly used to detect objects in an image are image pyramids (Pang et al., 2019) and sliding windows (Blue and Brindha, 2020). On the other hand, they have two main drawbacks: i) time consuming due to the looping process and ii) different detection rates as a result of applying parameters with different values. The main idea of the region proposal algorithm (RPA) (Taghizadeh and Chalechale, 2020) is that it can inspect the image to define the regions, where an object is most likely to be located.
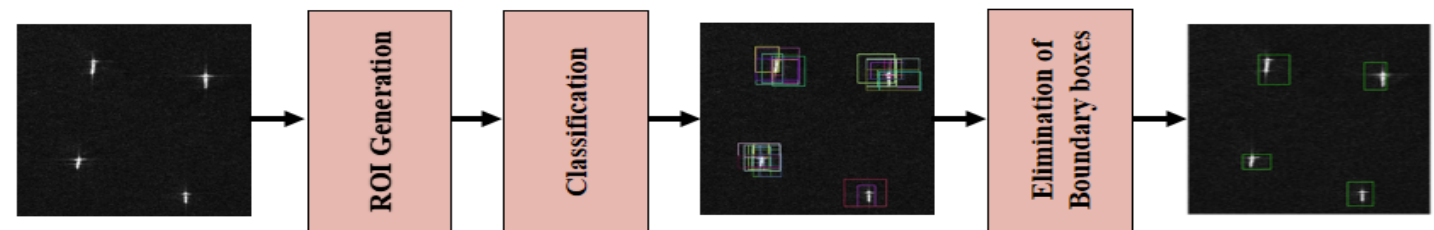

Figure 1: General flowchart of ship detection process in SAR images (Pang et al., 2019) 

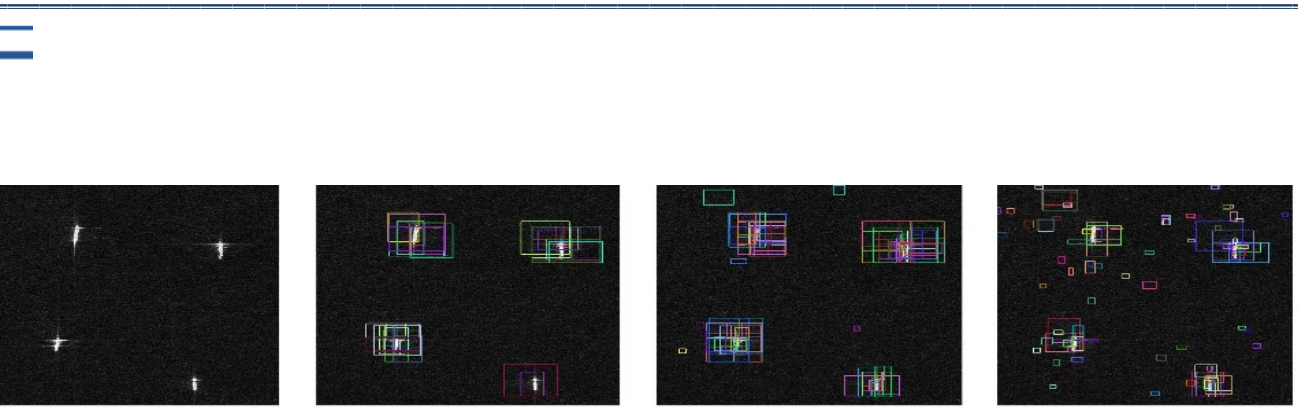

Figure 2: Sample generated ROIs using RPA
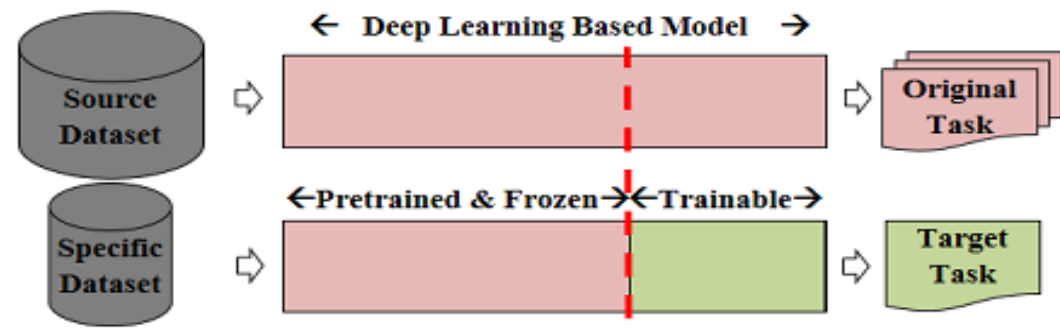

Figure 3: A typical transfer learning technique (Zhuang et al., 2020)

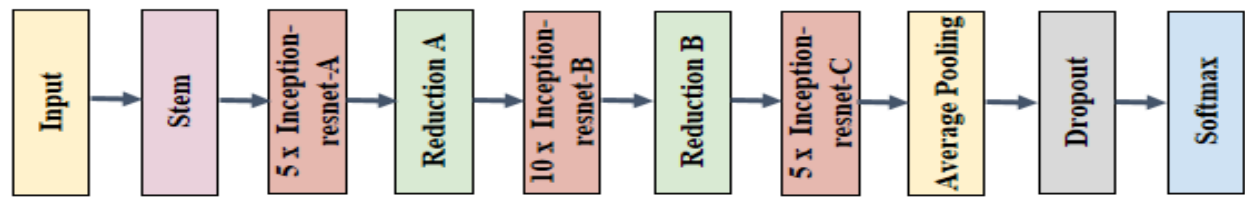

Figure 4: The basic network architecture of Inception-Resnet-v2 (Szegedy et al., 2017)

This can be achieved by using superpixel algorithm (Shang et al., 2020) for over-segmenting the input image. The RPA is considered to be more efficient than the traditional object detection techniques, i.e., image pyramids and sliding windows for some reasons as: i) the count of generated ROIs is few, ii) it is faster than exhaustively examining every scale/location of the input image, and iii) the amount of accuracy lost is minimal. In Figure 2, some samples, of the generated ROIs using the RPA, are shown, where white pixels represents the objects that may be classified as ships and colored boxes represent detected objects with different spatial resolutions that should be classified later as ships or not.

\subsection{The Proposed Hybrid DL Approach for Classification Step}

\subsubsection{The transfer learning technique}

In the scope of DL, transfer learning is performed by training a CNN classification model for a certain task using a large enough dataset or applying finetuning process to a pre-trained model, i.e., base model, using the new dataset targeted to the new task (Zhuang et al., 2020), as illustrated in Figure 3.

\subsubsection{Using the LSTM model}

The Long Short-Term Memory (LSTM) model is introduced as a special type and an enhanced version of recurrent neural networks (RNNs) to
There are a variety set of state-of-the-art CNN models that can be implemented as base-models for transfer learning such as VGG16 (Simonyan, and Zisserman, 2014), VGG19 (Wen et al., 2019), MobileNet V2 (Sandler et al., 2018), Xception (Chollet, 2017), Inception V2 (Alamsyah and Fachrurrozi, 2019), Inception-Resnet-V2 (Szegedy et al., 2017) and more.

In the proposed DL approach, Inception-ResnetV2 (Szegedy et al., 2017 and Wang et al., 2019), is utilized as the base model for transfer learning. InceptionResnet-V2 (Szegedy et al., 2017) is a CNN-based classification model trained on a large scale and available ImageNet dataset (Deng et al., 2009). InceptionResnet-V2, shown in Figure 4, is a combined structure of both Inception networks and ResNet networks. Inception networks have their own structure, that depends on convolutional filters of different sizes with pooling process within one layer, while ResNet networks utilize a shortcut connection structure which is flexible and dependent on the tasks due to its ability to skip one or more layers to avoid degradation as a result of deep structure and accelerate the training process as well.

solve the vanishing and exploding gradient problem (Wang et al., 2019). The LSTM model utilizes base memory cells $(\mathrm{mc})$ with a cell state and three gates: input, output, and forget gate, in each mc inside the hidden layer. These applied gates allow the LSTM 
model to efficiently remember and learn patterns in long-term dependencies. While CNN-based model has the ability to extract important features from the internal representation of the image, the LSTM model has the capacity to selectively remember patterns for a long duration of time in the target image. The LSTM models support different schemes, i.e., many-to-many scheme, in which a sequence of predicted output values can be generated by a sequence of input at a time. Moreover, stacking LSTM hidden layers are supposed to enhance the prediction efficiency and a count of two-layer stacked LSTM, as shown in Figure 5, is recommended to avoid the degradation problem (Deng et al., 2009).

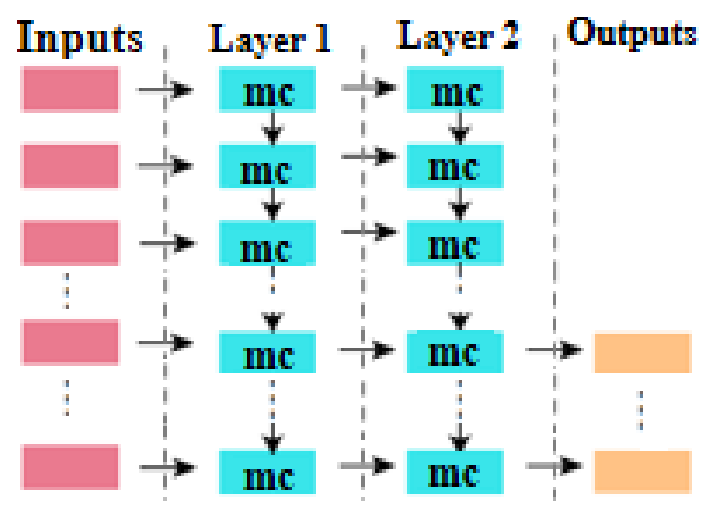

Figure 5: General structure of a two-layer stacked LSTM (Mahmoud et al., 2021)

In the classification step of the ship detection process, the LSTM model based on many-to-many scheme, in a two-layer stacked version, is combined with the fine-tuned Inception Resnet-V2-base model, in a hybrid classification DL-based model. The extracted features, from the Inception-ResnetV2- base model, will be enhanced with those predictions generated from the LSTM model in order to ultimately improve the classification accuracy, hence the whole detection process.

\subsubsection{Approaches of CNN and LSTM in a hybrid model}

This study proposes the pre-trained InceptionResnet-V2 as a base model for classification step. Two different approaches are suggested for combining the two-layer stacked LSTM model with the Inception-Resnet-V2 base model in a proposed hybrid model. The first hybrid model implements the pre-trained Inception-Resnet-V2 and the two- layer stacked LSTM in a parallel form, while the second approach implements them in a cascaded one. The input size for all approaches are $224 * 224 * 3$ that accommodates all ship sizes in the data set, as shown in Table 1, which are ranged from $7 * 7$ to $211 * 298$ in SAR images of size $500 * 500$ in average spatial resolution.

In the first suggested hybrid model, i.e., the proposed parallel model as shown in Figure 6, the input to the LSTM branch is first transferred to a gray scale, i.e., $224 * 224$. Then, each gray scale input is reshaped into 28 batches, each is of size 1792, and fed to the LSTM branch batch by batch. The extracted features from the pre-trained Inception-Resnet-V2 of dimension $5 * 5 * 1536$ are merged by those predictions form the two-layer stacked LSTM of dimension 1536 through elementwise multiplication with each batch of the 25 batches output from Inception-ResnetV2. The output of the 25 element-wise multiplications with the batch of dimension 1536 from LSTM is flattened in a merge layer of enhanced extracted features of dimension 38400. The enhanced extracted features, from the merged layer, are fed as input to a classification phase consisting of two cascaded fully connected layers of size 256 and 128 , respectively followed by a Softmax output layer, as shown in Figure 7.

In the second hybrid model, i.e., the proposed cascaded model shown in Figure 8, the extracted features, from the pretrained Inception-Resnet-V2 of dimension $5 * 5 * 1536$, are reshaped to form 25 timed series batches, each batch of size 1536. The reshaped batches are then fed as 25 input sequences to the LSTM model that generates 25 batches of predicted values, each batch of size 512. The 25 predicted batches are reshaped to form enhanced extracted features of dimension 12800 in the merge layer. The merged enhanced features are directed to two fully connected layers of size 256 and 128, respectively, followed by the Softmax function as illustrated in Figure 9.

\subsubsection{Overlapped bounding-boxes elimination using non-maxima suppression}

Extra bounding-boxes around the same detected object can be eliminated by applying a Non-Maxima Suppression (NMS) (Hosang et al., 2017) that can collapse those weak bounding-boxes based on a given confidence. 


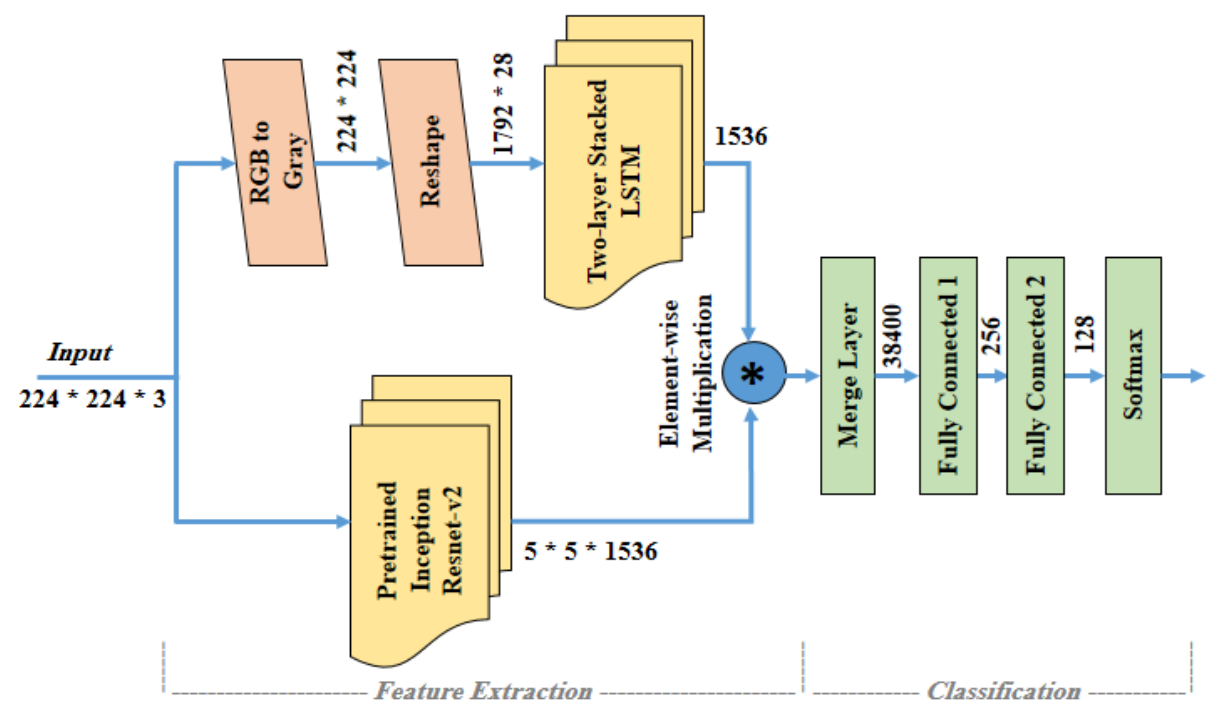

Figure 6: The parallel form structure of the proposed hybrid model

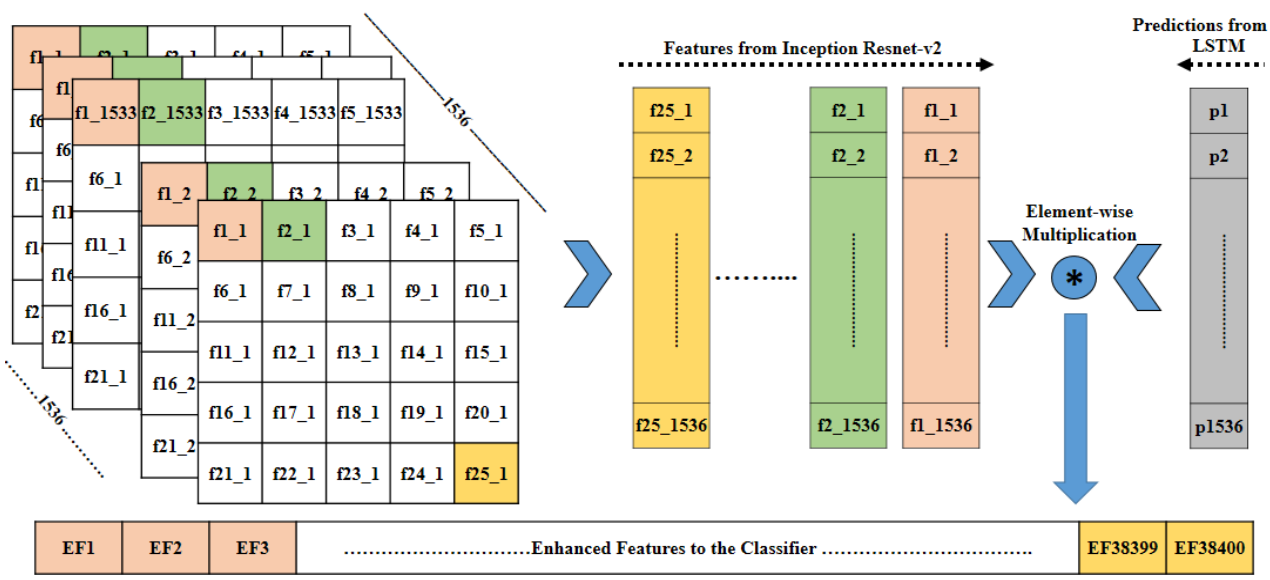

Figure 7: Enhancing the extracted features in the proposed parallel model

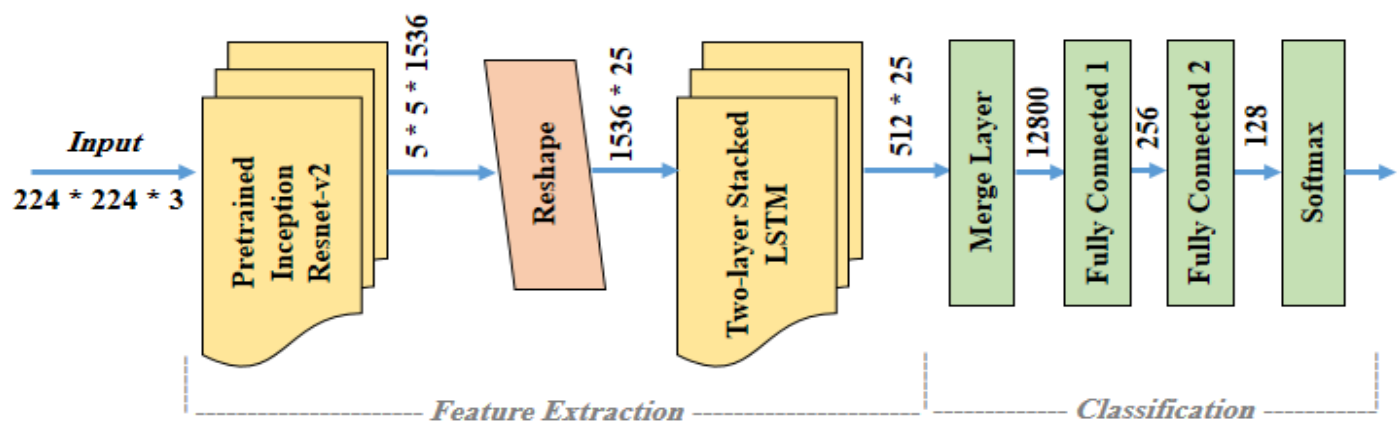

Figure 8: The cascaded form structure of the proposed hybrid model 

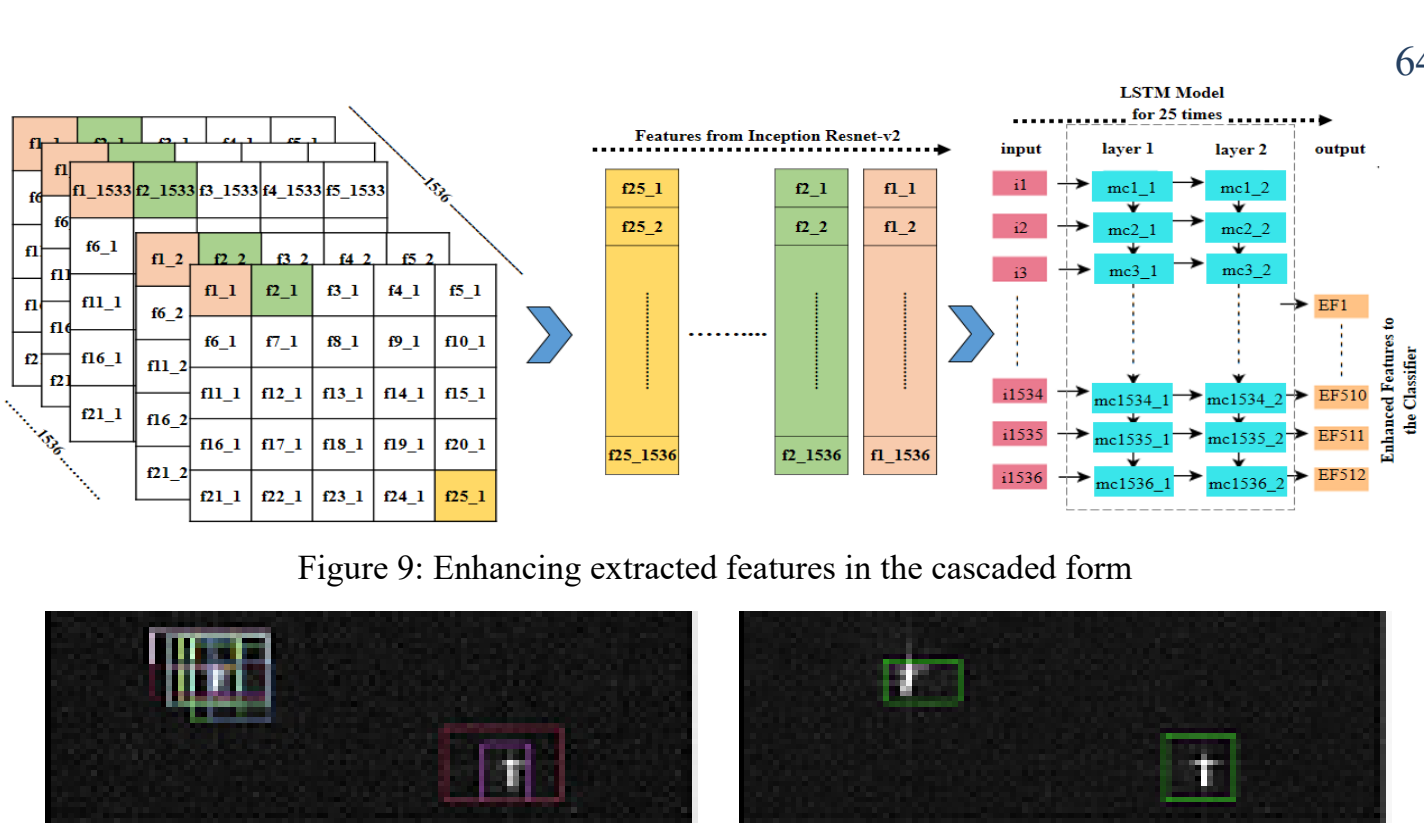

Figure 9: Enhancing extracted features in the cascaded form
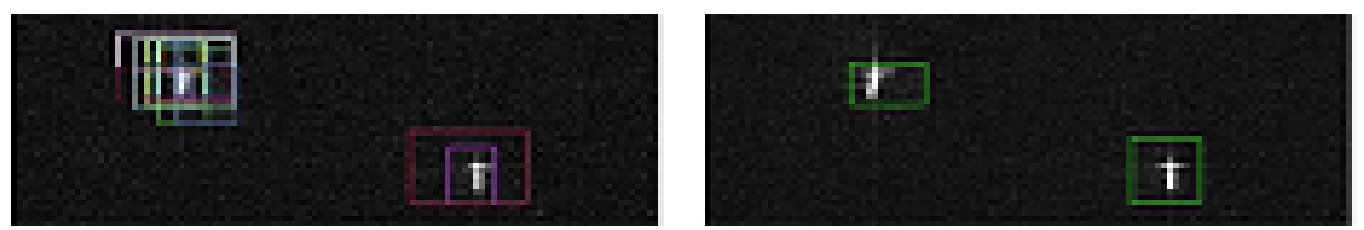

Figure 10: Bounding-boxes (a) before and (b) after applying NMS on the overlapped boxes

Table 1: Full description of the open (SSDD) (Li et al., 2017)

\begin{tabular}{|l|l|}
\hline Sensors & Sentinel-1, RadarSat-2, TerraSAR-X \\
\hline Place & Visakhapatnam, India; Yantai, China \\
\hline Polarization & $\mathrm{HH}, \mathrm{VV}, \mathrm{HV}, \mathrm{VH}$ \\
\hline Resolution & $1 \mathrm{~m}-10 \mathrm{~m}$ \\
\hline Scene & Inshore, offshore \\
\hline Num. of images & 1,160 \\
\hline Avg. size of Images & $500 \times 500$ \\
\hline Num. of ships & 2,358 \\
\hline Avg. Num. of Ships per image & 2.03 \\
\hline Smallest ships size & $7 \times 7$ \\
\hline Biggest ships size & $211 \times 298$ \\
\hline
\end{tabular}

The benefit of using NMS is to remove all the overlapped boxes except only one that perfectly surrounds the detected object hence; obtain only one detection per object. NMS algorithm starts by choosing the boundary box with the highest confidence score and suggests that it perfectly bounds the object, while the other boundary boxes, for that object, are suppressed. For the rest boundary boxes the next one with the highest confidence score is selected again and the whole process is repeated till finishing all the remaining boundary boxes. Figure 10 shows the difference between the output image before and after using the NMS algorithm.

\section{Experimental Work}

\subsection{Dataset Description}

The open SAR Ship Detection Dataset (SSDD) (Li et al., 2017) is used to verify the effectiveness of the proposed model for ship detection through training, validation and testing phases. SSDD is proposed in 2017 and already has been widely used in researches as in (Cui et al., 2019, Chang et al., 2019, Zhang et al., 2019(a), Zhang and Zhang, 2019 and Zhang et al., 2019(b). SSDD contains 1160 SAR images with a total of 2358 ships. Images of SSDD have different resolutions and polarization modes that effectively allows to evaluate the robustness of the proposed ship detection approach. A full description of SSDD is shown in Table 1.

\subsection{Training Strategy}

SSDD is randomly divided into $70 \%$ for training, $10 \%$ for validation and $20 \%$ for testing. The training and validation sets are pre-processed by count of 9 augmentation techniques: 1) horizontal shift, 2) vertical shift, 3) horizontal-vertical shift, 4) horizontal flip, 5) vertical flip, 6) random rotation, 7) random brightness, 8) random zoom, and 9) Gaussian noise, to generate new image patches that is divided into two classes ship and scene. In experiments, the learning rate used is 0.0001 and the number of epochs is 100 . The batch size used is 32 
and the model utilizes Adam optimizer (Ogaltsov and Tyurin, 2019). The workstation used has 10th generation Intel core I7-10700T processor with 16 GB RAM and an ASUS Geforce GTX 1650, 1733 $\mathrm{MHz}, 2560$ CUDA cores with $6 \mathrm{~GB}$ dedicated memory GPU. The environmental software includes Python 3.6.3, Tensorflow 1.7.0, Keras 2.1.2, CUDA 9.1 and cudnn 7.1.2. After the training and validation phases, i.e., in the test phase, the proposed DL model is fed with ROIs generated from test images using the RPA for classification purposes. Finally, the NMS algorithm will be applied to eliminate the unnecessary overlapped bounding-boxes in the ROIs of classified ships and then generates the final output SAR image from the input one, with non-overlapped bounding-boxes around the detected ships.

\subsection{Evaluation Metrics}

The most commonly used metrics for evaluating deep learningbased object detectors are Recall, Precision and mean Average Precision ( $m A P$ ). These metrics are defined by:

$$
\begin{aligned}
& \text { Recall }=T P /(T P+F N) \\
& \text { Precision }=T P /(T P+F P) \\
& m A P=\int_{0}^{1} P(R) d R
\end{aligned}
$$

Equation 3

where $T P$ is True Positive rate, $T N$ is True Negative rate, $F N$ is False Negative rate, $F P$ is False Positive rate, $P$ is Precision, $R$ is the Recall, $P(R)$ is Precision-Recall $(P-R)$ curve, by which $m A P$ is used to represent detection accuracy. Precision is the ratio between the True Positives and all the Positives. For the target problem statement, it is a measure of correctly identified objects as ships out of all detected ships. It reflects how reproducible predictions are, even if they are far from the accepted value, i.e., the reliability of the system. Recall is the measure of the proposed model correctly identifying True Positives. Thus, for all existing ships in the scene, recall reflects how many of them are correctly identified as ships, i.e., it gives a measure of how accurately the model is able to identify the relevant data. Recall reflects the sensitivity of the model. So, the model with higher recall is more sensitive to ships and can accurately detect most of ships in the input scenes regardless its scale or location. Precision-Recall Curve $P(R)$, is a direct representation of the Precision(y-axis) and the Recall(x-axis). Precision-Recall curves summarize the trade-off between the true positive rate and the positive predictive value for a predictive model using different probability thresholds.

\section{Results and Discussion}

Experimental results of the proposed parallel model shows an accuracy of $94 \%$, while the proposed cascaded model shows an accuracy of $97 \%$, as shown in their ROC curves and the associated AUCs in Figure 11. It is concluded that the cascaded form of the proposed hybrid model outperforms the parallel form in terms of classification accuracy by a ratio of $3.19 \%$.

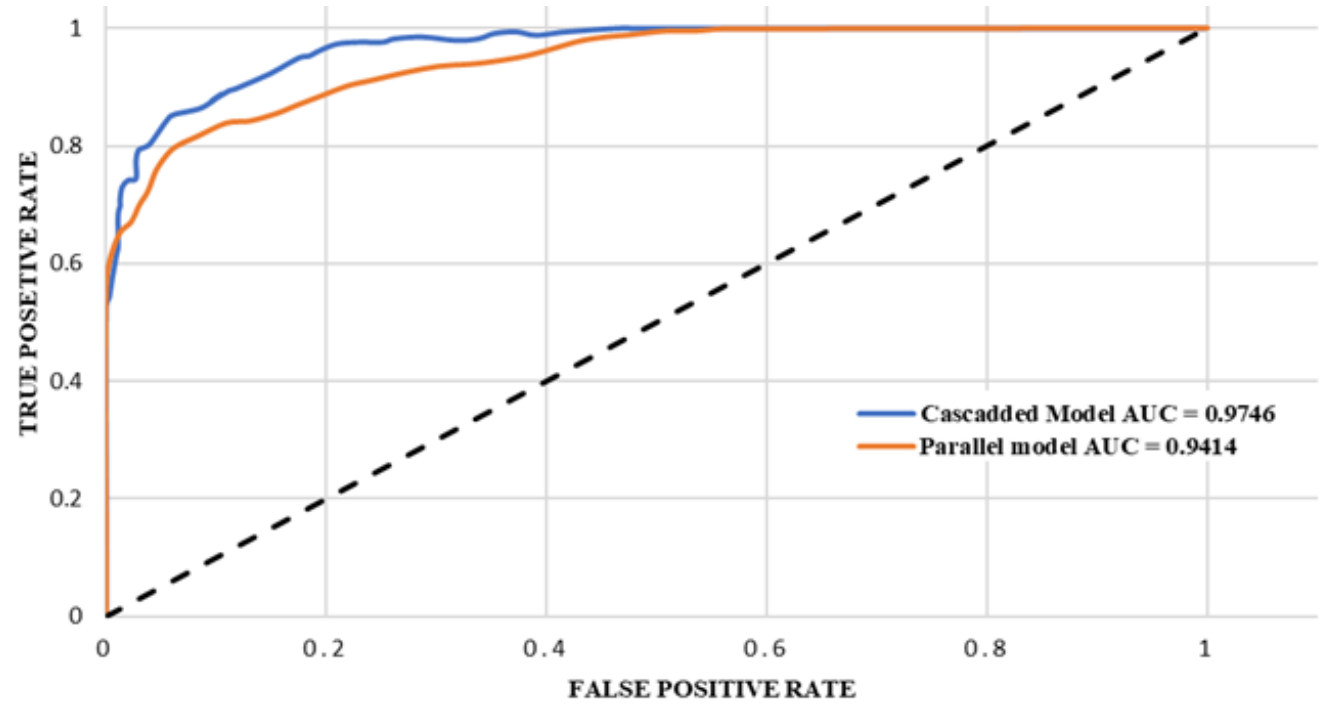

Figure 11: The ROC curve of the classification module 


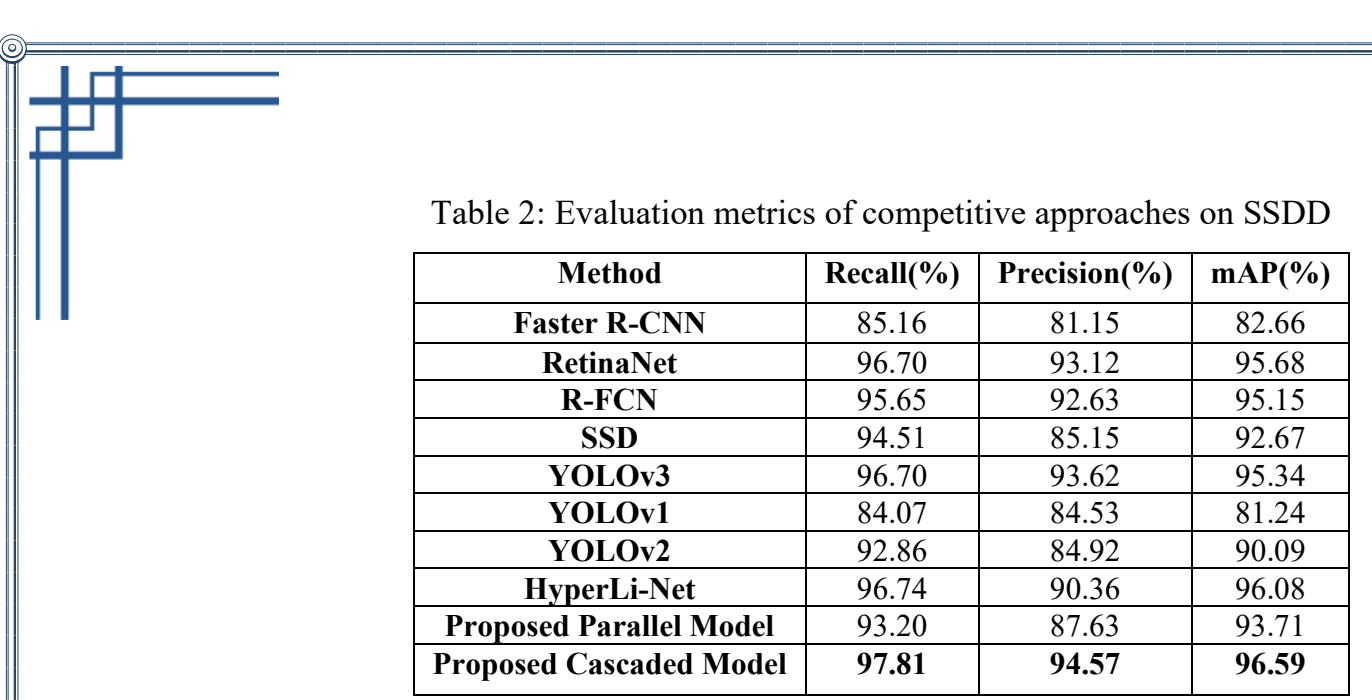

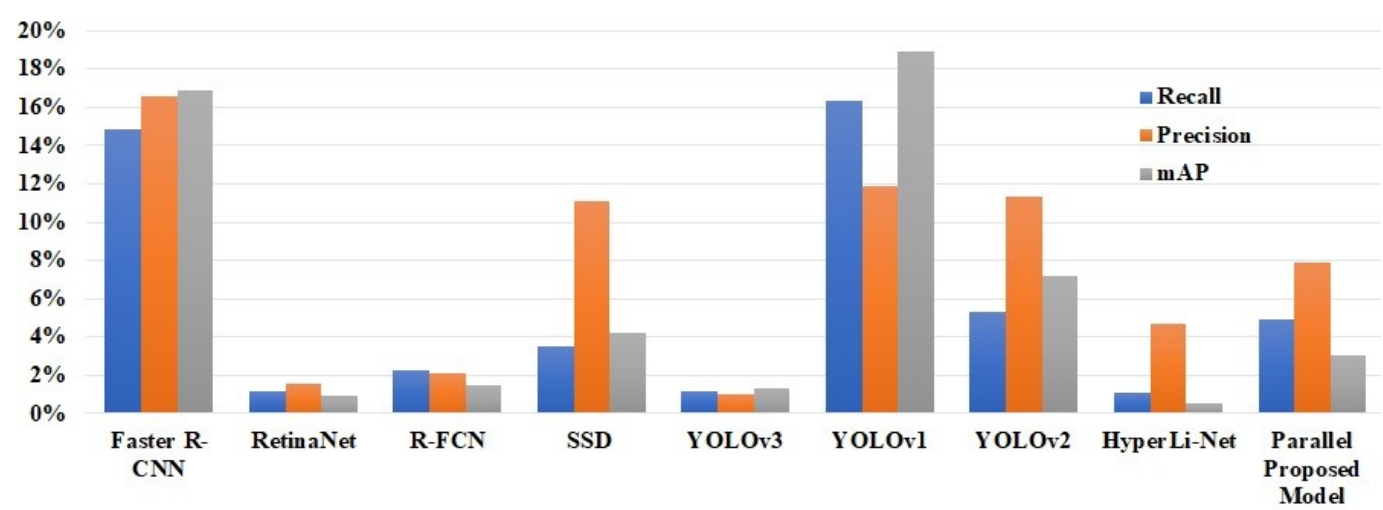

Figure 12: Improvement ratios (\%) in recall, precision and mAP of the proposed cascaded model as opposed to the competing approaches

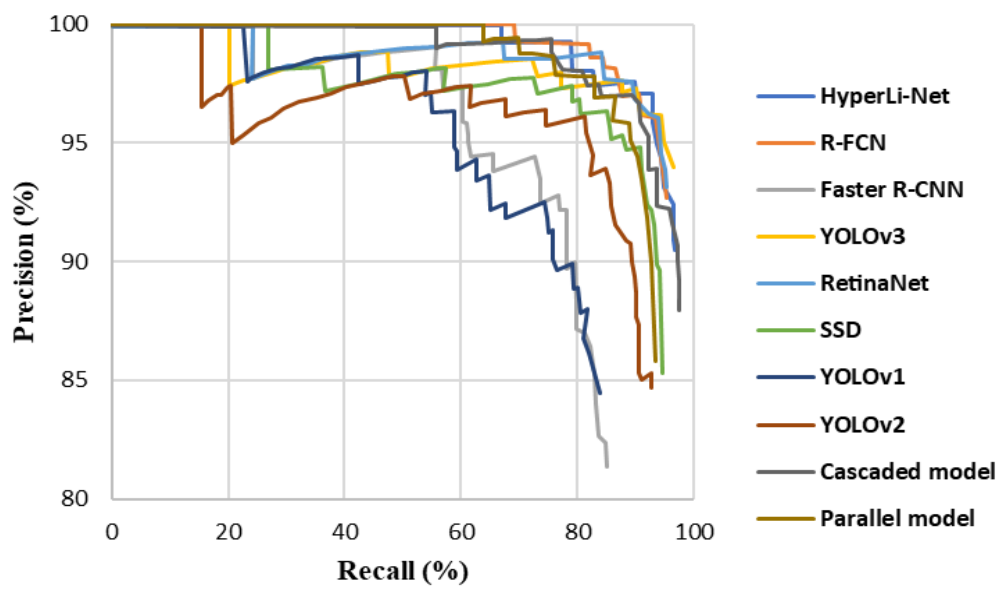

Figure 13: Precision-Recall (P-R) curves of all competing approaches

The two proposed hybrid models, in parallel and cascaded form separately, are compared with the most recent state-of-the-art approaches that utilize the same dataset for system training and evaluation (SSDD). The competitive approaches are YOLOv1 (Redmon et al., Wang), YOLOv3 (Redmon and Farhadi, 2018), YOLOv2 (Redmon and Farhadi, 2017), R-FCN (Dai et al., 2016), RetinaNet (Lin et al., 2017), Faster R-CNN (Ren et al., 2016), SSD (Liu et al., 2016) and HyperLi-Net (Zhang et al., 


Table 3: Evaluation metrics of competitive approaches on inshore and offshore scenes of SSDD
\begin{tabular}{|l|c|c|c|c|c|c|}
\hline \hline \multirow{2}{*}{ Method } & \multicolumn{3}{c|}{ Inshore } \\
\cline { 2 - 8 } & Recall & Precision & mAP & Recall & Precision & mAP \\
\hline & 70.35 & 62.05 & 66.22 & 98.12 & 94.32 & 97.68 \\
\hline Faster R-CNN & 47.67 & 87.23 & 46.52 & 79.57 & 99.33 & 79.38 \\
\hline SSD & 48.26 & 83.00 & 45.66 & 83.60 & 97.80 & 83.35 \\
\hline RetinaNet & 85.40 & 73.30 & 84.10 & 96.40 & 96.40 & 98.20 \\
\hline Attention RPN & 66.71 & 79.30 & 43.28 & 80.07 & 98.34 & 81.815 \\
\hline Proposed Parallel Model & 86.70 & 88.55 & 85.51 & 99.13 & 99.57 & 99.36 \\
\hline
\end{tabular}

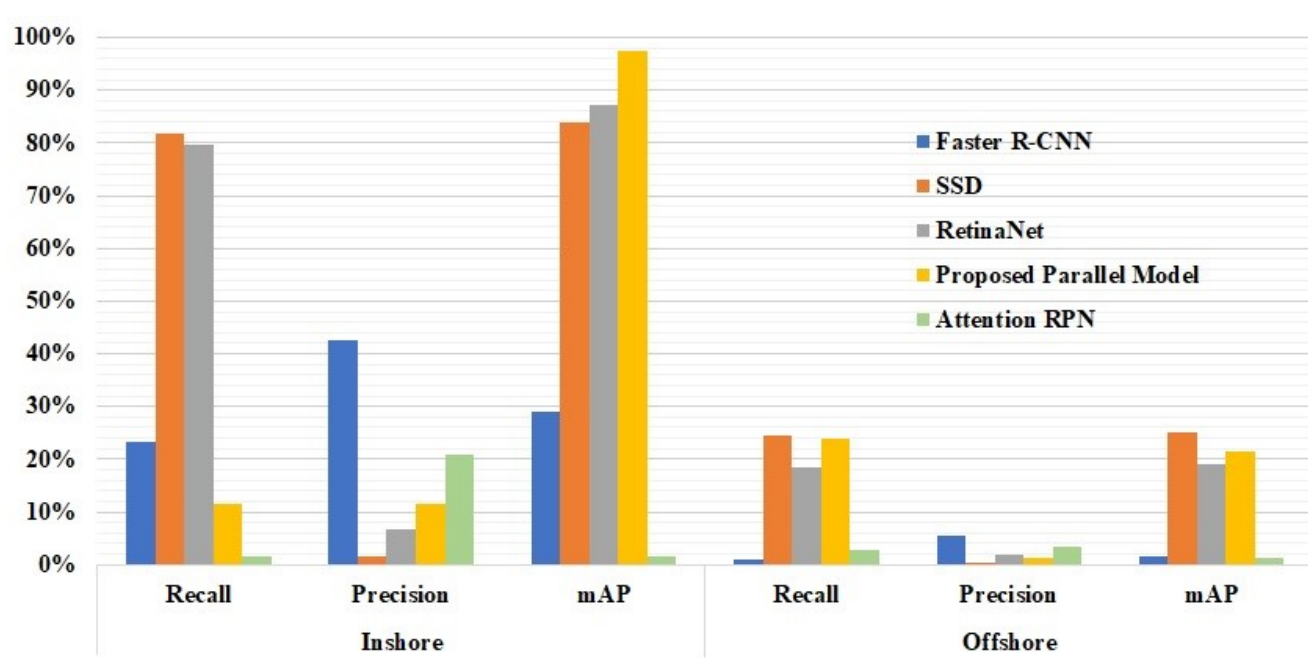

Figure 14: Improvement ratios (\%) in recall, precision and mAP of the proposed cascaded model for inshore and offshore cases
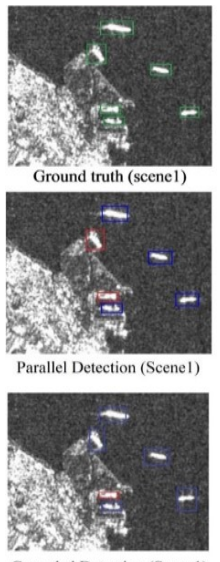

Cascaded Detection (Scene)

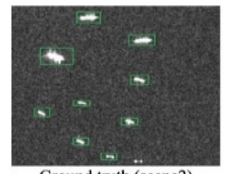

Ground truth (scene2)
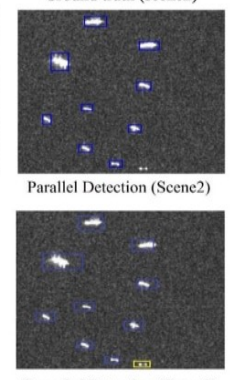

Cascaded Detection (Scene2)
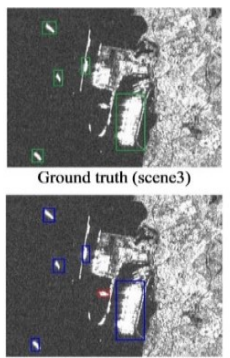

Parallel Detection (Scene3)

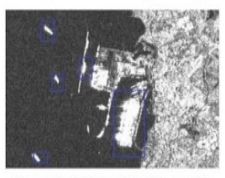

Cascaded Detection (Scene3)

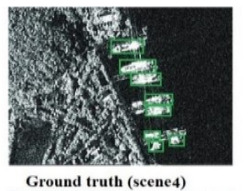

Ground truth (scene4)

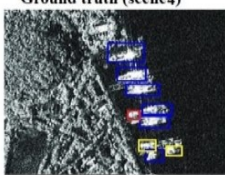

Parallel Detection (Scene 4)

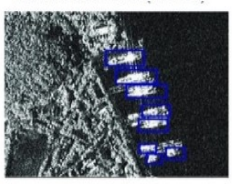

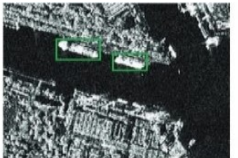

Ground truth (scene5)

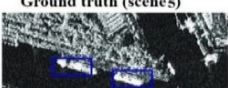

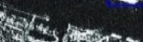

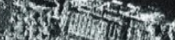

Hexis

Parallel Detection (Seene5)

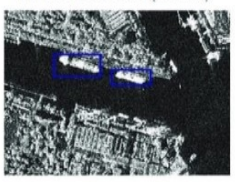

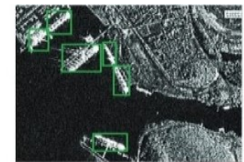

Ground truth (scene 6)

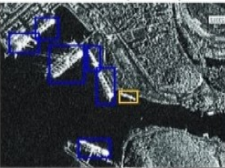

Parallel Detection (Scene6)

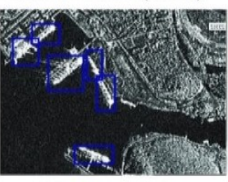

Figure 15: Different test scenes for both the proposed parallel and cascaded models, where blue boundingboxes means correct detection, red means missed detection, and yellow means false-alarms

A more challenged capability of any ship detection process is its ability to reliably detect ships in the SAR images regardless their sizes or locations, i.e., inshore or offshore. Table 3 presents the performance of the proposed cascaded model in detecting ships in both inshore and offshore scenes. As shown in Figure 14, for the inshore cases, the proposed cascaded model shows high efficiency by enhancement ratios up to $81.88 \%, 42.71 \%$ and $97.55 \%$ in terms of Recall, Precision and mAP, respectively relative to the competing state-of-theart approaches. Meanwhile, for the offshore cases, the proposed cascaded model can achieve enhancements up to $24.58 \%, 5.57 \%$ and $25.17 \%$, 
respectively for the same metrics. The improvements achieved by the proposed cascaded model in the recall and precision metrics for both inshore and offshore denote a higher combination of sensitivity and reliability of the proposed model in the challenged cases, especially, i.e., inshore ones.

Different output samples (ground truth and detected ships) in different scenes, from both the proposed parallel model and the proposed cascaded model, are shown in Figure 15.

\section{Conclusions and Future Work}

This paper presents two deep learning hybrid approaches, combining the pre-trained InceptionResent-V2 and a two-layer stacked LSTM, for ship classification in a three-step detection process in SAR images. The first proposed approach, generated by the bagging method, combines them in parallel form, while the other, generated by the boosting method, combines them in the cascaded form. In detection process the region proposal algorithm is applied for ROI generations, while NonMaxima algorithm. is implemented for eliminating overlapped bounding-boxes.

Both models are trained using SSDD dataset and evaluated using Recall, Precision and mAP evaluation metrics. Experimental results show that the proposed cascaded model outperforms all competitive state-of-the-art approaches, including the parallel one, with ratios of $97.81 \%, 94.57 \%$, and $96.59 \%$ for recall, precision, and mean average precision respectively. Moreover, the results shows an outstanding efficiency of the proposed cascaded model especially for inshore scenes as challenged cases, as it achieves $81.88 \%, 42.71 \%$ and $97.55 \%$ for the same metrics respectively. The outstanding results show that the proposed cascaded model capable of performing multiple ship detection in SAR images with relatively high reliability regardless their sizes or locations, i.e., high sensitivity model.

The future work is suggested to be an enhanced version of the proposed model that has a better ship detection accuracy for both inshore and offshore scenes. It is intended to apply attention-based techniques, which boost the model to higher detection accuracy. Relative to the practical implementation, the future work will be focused on studying practical implementation of such deep learning applications, as intensive codes, on embedded platforms, i.e., on-board computers of the aerospace vehicles such as Unmanned Aerial Vehicle (UAVs) and satellites (Elshazly et al., 2021). These implementations will be tested in different environments: as software on embedded micro-controllers, or hardware implementation on FPGA and even on both as a hardware and software co-design. The future work will focus on optimizing these deep learning based models, as high computational applications, to work on different onboard embedded platforms including hardware and/or software partitioning, code analysis, code transformation, and code re-targeting heterogeneous computing platforms.

\section{References}

Ai, J., Qi, X., Yu, W., Deng, Y., Liu, F. and Shi, L., 2010, A New CFAR Ship Detection Algorithm Based on 2-D Joint Log-Normal Distribution in SAR Images. IEEE Geosci. Remote Sens. Lett., Vol. 7(4), 806-810.

Alamsyah, D. and Fachrurrozi, M., 2019, Faster rcnn with Inception v2 for Fingertip Detection in Homogenous Background Image. IOP J. of Physics: Conf. Series, Vol. 1196, doi: 10.1088/1742-6596/1196/1/012017.

Arivazhagan, S., Lilly Jebarani, W. S., Newlin Shebiah, R., Ligi, S. V., Hareesh Kumar, P. and Anilkumar, K., 2019, Significance Based Ship Detection from Sar Imagery. $1^{\text {st }}$ International Conference on Innovations in Information and Communication Technology (ICIICT), 1-5, doi:10.1109/ICIICT1.2019.8741483.

Blue, S. T. and Brindha, M., 2020, An Edge Detection and Sliding Window Based Approach to Improve Object Localization in yolov3. Intern. Conf. on Machine Learning, Image Processing, Network Security and Data Sciences. Springer, 160-174, doi: 10.1007/978981-15-6315-7 13.

Carman, T. and Kolhatkar, A., 2020, A Comparison of Fixed Threshold CFAR and CNN Ship Detection Methods for S-band NovaSAR Images. The $34^{\text {th }}$ Annual Small Satellite Conference. https://digitalcommons.usu.edu/smallsat/2020/all2020/162/.

Chang, Y., Anagaw, A., Chang, L., Wang, Y., Hsiao, C. and Lee, W., 2019, Ship Detection Based on YOLOv2 for SAR Imagery. Remote Sensing, Vol. 11(7), 1-14, doi: 10.3390/rs11070786.

Chaturvedi, S. K., 2019, Study of Synthetic Aperture Radar and Automatic Identification System for Ship Target Detection. Journal of Ocean Engineering and Science, Vol. 4(2), 173182, doi:10.1016/j.joes.2019.04.002.

Chollet, F., 2017, Xception: Deep Learning with Depthwise Separable Convolutions. The IEEE 
Conf. on Comp. Vision and Patt. Recog., 12511258, doi: 10.1109 / CVPR.2017.195.

Cui, Z., Li, Q., Cao, Z. and Liu, N., 2019, Dense Attention Pyramid Networks for Multi-Scale Ship Detection in SAR Images. IEEE Trans. on Geoscience and Remote Sensing, Vol. 57(11), 8983-8997, doi: 10.1109 /TGRS. 2019.2923988.

Cui, Z., Wang, X., Liu, N., Cao, Z. and Yang, J., 2021, Ship Detection in Large-Scale Sar Images Via Spatial Shuffle-Group Enhance Attention. IEEE Transactions on Geoscience and Remote Sensing, Vol. 59(1), 379-391, doi:10.1109/TGRS-.2020.2997200.

Dai, J., Li, Y., He, K. and Sun, J., 2016, R-FCN: Object Detection Via Region-Based Fully Convolutional Networks. Proceedings of the 30 th International Conference on Neural Information Processing Systems, 379-387, arXiv preprint arXiv:1605.06409.

Deng, J., Dong, W., Socher, R., Li, L., Li, K. and Fei, L., 2009, Imagenet: A Large-Scale Hierarchical Image Database. IEEE Conf. on Comp. Vision and Patt. Recog., 248-255, doi: 10.1109 /CVPR. 2009.5206848.

Elshazly, A., Elliethy, A. and Elshafey, M. A., 2021, Tactics Overview for Implementing High Performance Computing on Embedded Platforms. IOP Conf. Ser.: Mater. Sci. Eng., Vol. 1172(1), doi: 10.1088/1757-899x/1172/1/012034.

Fouad, M. M., Mostafa, E. M. and Elshafey, M. A., 2020, Detection and Localization Enhancement for Satellite Images with Small Forgeries using Modified GAN-based CNN Structure. Intern. J. of Advances in Intelligent Informatics, Vol. 6(3), 278-289, doi: 10.26555 /ijain.v 6i3.548.

Hosang, J., Benenson, R. and Schiele, B., 2017, Learning Nonmaximum Suppression. The IEEE Conf. on Comp. Vision and Patt. Recog., 45074515, doi: 10.1109 / CVPR.2017.685.

Hou, B., Yang, W., Wang, S. and Hou, X., 2013, SAR Image Ship Detection Based on Visual Attention Model. Proc. IEEE Int. Geosci. Remote Sens. Symp. - IGARSS, 2003-2006.

Kang, M., Leng, X., Lin, Z. and Ji, K., 2017, A Modified Faster RCNN Based on CFAR Algorithm for SAR Ship Detection. IEEE Intern. W. on Remote Sensing with Intelligent Proc., 14, doi: 10.1109 /RSIP. 2017.7958815.

Li, J., Qu, C. and Shao, J., 2017, Ship Detection in SAR Images Based on an Improved Faster RCNN. IEEE SAR in Big Data Era: Models, Methods and App., 1-6, doi: 10.1109 /BIGSARDATA. 2017.8124934.
Liang, Y., Sun, K., Zeng, Y., Li, G. and Xing, M., 2020, An Adaptive Hierarchical Detection Method for Ship Targets in High-Resolution SAR Images. Remote Sens., Vol. 12(2), 1-22, https://doi.org/10.3390/rs12020303.

Liu, W., Anguelov, D., Erhan, D., Szegedy, C., Reed, S., Fu, C. and Berg, A., 2016, SSD: Single Shot Multibox Detector. European Conf. on Comp. Vision, 21-37, doi: 10.1007/978-3-31946448-0 2.

Lin, T., Goyal, P., Girshick, R., He, K. and Dollar, P., 2017, Focal Loss for Dense Object Detection. IEEE Intern. Conf. on Comp. Vision, 2980 2988, doi: 10.1109/TPAMI. 2018.2858826.

Liu, N., Cao, Z., Cui, Z., Pi, Y. and Dang, S., 2019, Multiscale Proposal Generation for Ship Detection in SAR Images. Remote Sensing, Vol. 11(5), doi:10.3390/rs11050526.

Mahmoud, T. A., Shehab, A. F. and Elshafey, M. A., 2021, Different Long Short-Term Memory Approaches to Enhance Prediction-Based Satellite Telemetry Compression. Journal of Aerospace Information Systems, 1-8, doi: 10.2514/1.I010906.

Ogaltsov, A. and Tyurin, A., 2019, Heuristic Adaptive Fast Gradient Method in Stochastic Optimization Tasks. arXiv: Optimization and Control, doi: 10.1134 / S0965542520070088.

Pang, Y., Wang, T., Anwer, R., Khan, F. and Shao, L., 2019, Efficient Featurized Image Pyramid Network for Single Shot Detector. Proc. of the IEEE Conf. on Comp. Vision and Patt. Recog., 7336-7344, doi: 10.1109 /CVPR. 2019.00751.

Redmon, J., Divvala, S., Girshick, R. and Farhadi, A., 2016, You Only Look Once: Unified, RealTime Object Detection. IEEE Conf. on Comp. Vision and Patt. Recog., 779-788, doi: 10.1109/CVPR.2016.91.

Redmon, J. and Farhadi, A., 2017, Yolo9000: Better, Faster, and Stronger. IEEE Conf. on Comp. Vision and Patt. Recog., 7263-7271, doi: 10.1109 /CVPR. 2017.690.

Redmon, J. and Farhadi, 2018, A., Yolov3: An Incremental Improvement. arXiv preprint arXiv: 1804.02767.

Ren, S., He, K., Girshick, R. and Sun, J., 2016, Faster R-CNN: Towards Real-Time Object Detection with Region Proposal Networks. IEEE Trans. on Patt. Ana. and Machine Intelli., Vol. 39(6), 1137-1149, doi: 10.1109 /TPAMI. 2016.2577031.

Sandler, M., Howard, A., Zhu, M., Zhmoginov, A. and Chen, L., 2018, Mobilenetv2: Inverted Residuals and Linear Bottlenecks. IEEE Conf. 
on Comp. Vision and Patt. Recog., 4510-4520, doi: 10.1109/CVPR.2018.00474.

Shang, R., Lin, J., Jiao, L., Yang, X. and Li, Y., 2020, Superpixel Boundary-Based Edge Description Algorithm for SAR Image Segmentation. IEEE J. of Selected Topics in Applied Earth Obs. and Remote Sensing, Vol. 13, 1971-1985, doi: 10.1109/JSTARS.2020.2987653.

Simonyan, K. and Zisserman, A., 2014, Very Deep Convolutional Networks for Large-Scale Image Recognition. arXiv preprint arXiv:1409.1556.

Song, S., Xu, B. and Yang, J., 2017, Ship Detection in Polarimetric SAR Images via Variational Bayesian Inference. IEEE J. of Selected Topics in Applied Earth Observations and Remote Sensing, Vol. 10(6), 2819-2829, doi: 10.1109/JSTARS.2017.2687473.

Szegedy, C., Ioffe, S., Vanhoucke, V. and Alemi, A., 2017, Inception-v4, Inception-Resnet and the Impact of Resid UAL Connections on Learning. AAAI'17: Proceedings of the Thirty-First AAAI Conference on Artificial Intelligence Vol. 31., 4278-4284,

Taghizadeh, M. and Chalechale, A., 2020, Region Proposal Generation: A Hierarchical Merging Similarity-Based Algorithm. IEEE Intern. Conf. on Machine Vision and Image Proc., 1-6, doi:10.1109/MVIP 49855.2020. 9116912.

Wang, Y. and Liu, H., 2012, A Hierarchical Ship Detection Scheme for High Resolution SAR Images. IEEE Trans. Geosci. Remote Sens., Vol. 50(10), 4173-4184.

Wang, R., Li, J., Duan, Y., Cao, H. and Zhao, Y., 2018a, Study on the Combined Application of CFAR and Deep Learning in Ship Detection. Journal of the Indian Society of Remote Sensing, Vol. 46(9), 1413-1421.

Wang, Y., Wang, C. and Zhang, H., 2018b, Combining a Single Shot Multibox Detector with Transfer Learning for Ship Detection Using Sentinel-1 SAR Images, Remote Sensing Letters, Vol. 9(8), 780-788, doi: 10.1080 2150704X.2018.1475770.

Wang, X., Qin, Y., Wang, Y., Xiang, S. and Chen, H., 2019a, Reltanh: An Activation Function With Vanishing Gradient Resistance for SAEBased DNNs and its Application to Rotating Machinery Fault Diagnosis. Neurocomputing, Vol. 363, 88-98, doi:10.1016/j.neucom.2019.07.017.

Wang, Y., Wang, C., Zhang, H., Dong, Y. and Wei, S., 2019b, Automatic ship Detection Based on
Retina Image Net Classification with Deep Convolutional Neural Networks Net using Multiresolution Gaofen-3 Imagery. Remote Sensing, Vol. 11(5), 531, doi: 10.3390/rs11050531.

Wen, J., Li, X. and Gao, L., 2019, A New Transfer Learning Based on VGG-19 Network for Fault

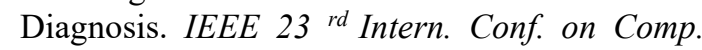
Supported Cooperative Work in Design, 205209, doi: 10.1109/CSCWD.2019. 8791884.

Zhang, X., Xiong, B., Dong, G. and Kuang, G., 2018, Ship Segmentation in SAR Images by Improved Nonlocal Active Contour Model. Sensors, Vol. 18(12), https://doi.org/10.3390/s18124220 p.4220.

Zhang, T. and Zhang, X., 2019, High-Speed Ship Detection inSAR Images Based on a Grid Convolutional Neural Network. Remote Sensing, Vol. 11(10), 1206, doi: 10.3390/rs11101206.

Zhang, T., Zhang, X., Shi, J. and Wei, S., 2019a, Depthwise Separable Convolution Neural Network for High-Speed SAR Ship Detection. Remote Sensing, Vol. 11(21), doi: $10.3390 /$ rs 11212483.

Zhang, X., Zhang, T., Shi, J. and Wei, S., 2019b, High-speed and High-Accurate SAR Ship Detection Based on a Depthwise Separable Convolution Neural Network. J. Radars, Vol. 8(6), 841-851, doi: 10.12000/JR19111.

Zhang, T., Zhang, X., Shi, J. and Wei, S., 2020, Hyperli-net: A Hyper-Light Deep Learning Network for High-Accurate and High-Speed Ship Detection from Synthetic Aperture Radar Imagery. ISPRS Journal of Photogrammetry and Remote Sensing, Vol. 167, 123-153, doi:10.1016 /j.isprsjprs.2020.05.016.

Zhao, Y., Zhao, L., Xiong, B. and Kuang, G., 2020, Attention Receptive Pyramid Network for Ship Detection in SAR Images. IEEE Journal of Selected Topics in Appl. Earth Obser. and Rem. Sensing, Vol. 13, 2738-2756 , doi: 10.1109 /JSTARS. 2020.2997081.

Zhou, Y., Cai, Z., Zhu, Y. and Yan, J., 2020, Automatic Ship Detection in SAR Image Based on Multi-Scale Faster RCNN. IOP J. of Physics: Conf. Series, Vol. 1550, doi: 10.1088/17426596/1550/4/042006.

Zhuang, F., Qi, Z., Duan, K., Xi, D., Zhu, Y., Zhu, H., Xiong, H. and He, Q., 2020, A Comprehensive Survey on Transfer Learning. Proc. of the IEEE, Vol. 109(1), 43-76, doi: 10.1109/JPROC.2020.3004555. 